\title{
Caracterización agrícola del Municipio de La Virtud, Departamento de Lempira
}

Jessica Gabriela Villatoro Escobar

\section{Resumen}

El presente trabajo tuvo el objetivo de caracterizar los tipos de agricultura y cultivos predominantes en el Municipio de La Virtud. Este se encuentra ubicado en el sur de Lempira con una superficie de 6822.1452 Ha y cuenta con afluentes que se unen a la Subcuenca de río Mocal.

Los pasos metodológicos realizados fueron: identificación de comunidades de estudio pertenecientes al Municipio de La Virtud, determinación de muestra, realización de encuesta a 40 productores de las comunidades de Tixila, la Trinidad y Solicor, caracterización y análisis de datos obtenidos de fuentes bibliográficas y encuestas. De los principales resultados obtenidos y analizados se determinó que la población rural del Municipio de la Virtud, siembra en mayor cantidad cultivos de maíz, frijol y sorgo. Por otro lado entre el año 1986 y 2009 se dio un aumento de la cobertura agrícola de la zona, sin embargo en la actualidad aún existen comunidades del Municipio de La Virtud que practican la agricultura tradicional, aunque perciben la agricultura Agroforestal como una buena práctica de cultivos. Se concluye que la metodología empleada permitió conocer como ha sido la situación pasada y actual de los tipos de agricultura empleadas por los productores del Municipio de La Virtud, misma que puede causar problemas en la cobertura de la tierra y en todo el medio ambiente. Con la caracterización obtenida del Municipio de La Virtud se procederá a una segunda etapa del estudio acerca de un análisis más exhaustivo que indique los cambios de cobertura agrícola vrs. Los tipos de agricultura empleadas.

Palabras Clave: Cobertura, cultivos, tipo de agricultura, Municipio, Caracterización

Jessica Gabriela Villatoro Escobar, (jessicavillatoro_escobar@yahoo.com) Departamento de Ciencia y Tecnologías de la Información Geográfica, Facultad de Ciencias Espaciales Universidad Nacional Autónoma de Honduras 


\section{Introducción}

La subcuenca del río Mocal se encuentra ubicada en el Departamento de Lempira, conformada por 119,004.919 Ha y rodeada por 18 Municipios. Esta Subcuenca constituye fuente importante de desarrollo para los ciudadanos que viven en Lempira por lo que se prevé algunos proyectos futuros, como ser pago/compensación por servicios ambientales.

Los cambios de cobertura agrícola y tipos de agricultura empleadas pueden repercutir sobre el agua de la subcuenca del río Mocal. , por lo tanto es necesario conocer el comportamiento de la cobertura agrícola en un periodo de tiempo seleccionado. Es así que el estudio está permitiendo adquirir información que sirva en primera instancia para caracterizar la zona, posteriormente para establecer escenarios de cambios de cobertura agrícola.

Actualmente los datos están siendo recolectados en 6 Municipios de Lempira (Belen Gualcho, San Manuel de Colohete, San Marcos de Caiquín, Tomalá, La Virtud y Candelaria), Aun están en proceso de recolección, sin embargo ya se cuenta con algunos resultados del Municipio de La Virtud.

Se plantea como objetivo del presente trabajo la caracterización de los tipos de agricultura y cultivos predominantes en el Municipio de La Virtud. Los resultados obtenidos constituirán información básica utilizable como paso previo y de apoyo para los resultados finales del estudio de Prospección de Cambios de Cobertura Agrícola en la Subcuenca del río Mocal, así mismo para estudios posteriores.

\section{Método}

El estudio realizado es de tipo exploratorio y se comenzó con la identificación de comunidades a encuestar, en este caso se encuestaron comunidades de Tixila, La trinidad y Solicor, ya que son las comunidades que tienen afluentes a la subcuenca del Río Mocal.

- Determinación de la Muestra

La muestra fue determinada utilizando la fórmula estadística que se observa a continuación: 


\section{Fórmulaemoleada}

$$
\mathrm{n}=\frac{\mathrm{n}_{\mathrm{o}}}{1+\frac{\mathrm{n}_{\mathrm{o}}}{\mathrm{N}}} \text { donde: } \mathrm{n}_{\mathrm{o}}=p^{*}(1-p)^{*}\left(\frac{\mathrm{z}\left(1-\frac{\alpha}{2}\right)}{\mathrm{d}}\right]^{2}
$$

Para realizar la formula se tomó en cuenta la población del área rural del Municipio de la Virtud, obteniendo la aplicación de 40 encuestas con un grado de confianza del $90 \%$.

\section{- Encuesta a productores}

Durante el mes de junio 2013 se llevaron a cabo encuestas a productores, solicitando básicamente que cultivos se siembran en la zona y el tipo de agricultura empleada, entre otros aspectos como prácticas agrícolas.

- Evaluación de las encuestas

Los datos obtenidos fueron digitados en el programa estadístico SPS y Excel, de lo cual se han obtenido algunas representaciones gráficas (histogramas). En tal sentido cabe mencionar que en la segunda etapa del estudio, se podrá reflejar la información Geográficamente a través de mapas elaborados en ArcGis, una vez recolectados más datos.

\section{- Caracterización}

A través de recuento bibliográfico, datos obtenidos de SINIT, INE y encuestas aplicadas, se ha realizado una breve descripción de la Zona de Estudio, a través de la Importancia Económica y potencialidad del sector agrícola, caracterización de tipo de agricultura, Clima, temperatura y precipitación.

\section{Resultados y Discusión}

- Localización

La virtud está ubicada en el Sur de Lempira, colinda al norte con el Municipio de Valladolid, Al Sur y Este con El Salvador y al Oeste con los Municipios de Gualcince y Mapulaca. (fig. 1) 


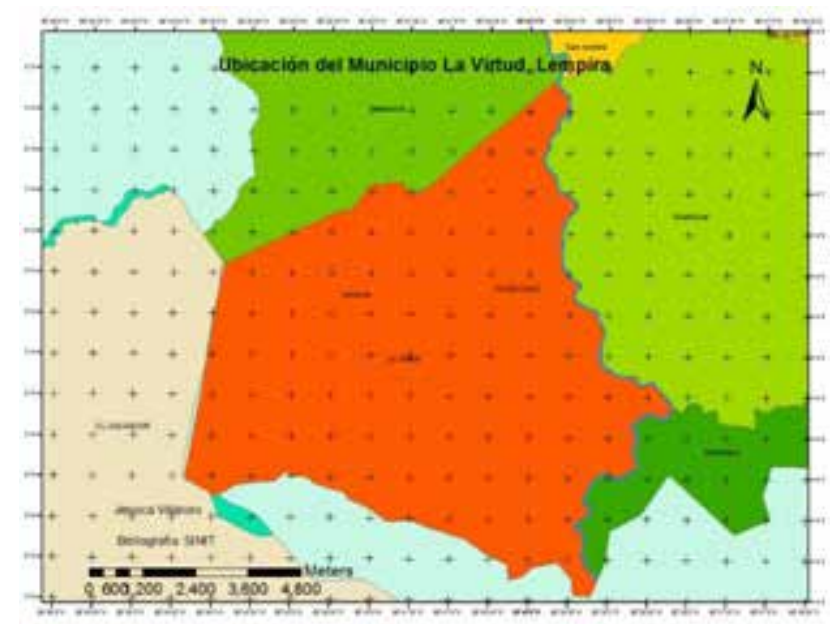

Figura 1. Localización del Municipio de La Virtud

- Importancia Económica y potencialidad del sector agrícola

El sector agrícola ha ejercido un papel importante en la economía de los Municipios de Lempira, ya que influyen sobre el PIB (producto interno bruto) del país. Ciertamente existen periodos de sequía a nivel nacional, las cuales afectan la agricultura, esto ha generado impactos negativos sobre la economía del Departamento de Lempira, en especial de los pequeños productores ubicados en zonas de ladera. (Fernandez, Liliana; Navarro, Edgardo; FAO, 2005) 
Indicador económico que refleja la implementación del sistema Quesungual mejorado

\begin{tabular}{|c|c|c|c|}
\hline Indieador & $\begin{array}{l}\text { Quema } \\
(\mathrm{N}=23)\end{array}$ & $\begin{array}{l}\text { Quema } \quad \text { con } \\
\text { químicos } \\
(\mathrm{N}=\mathbf{2 0})\end{array}$ & $\begin{array}{l}\text { Sistema } \\
\text { agroforestal } \\
\text { Quesungual } \\
(\mathrm{N}=30) \\
\end{array}$ \\
\hline $\begin{array}{l}\text { Producto Bruto } \\
\text { (Valor total } \\
\text { producción) ** }\end{array}$ & 3433.80 Lps. & $4561.22 \mathrm{Lps}$. & $5412.80 \mathrm{Lps}$. \\
\hline Maiz qq/mz ** & $14 q q$ & $26 \mathrm{qq}$ & $32 q q$ \\
\hline Maicillo $\mathrm{qq} / \mathrm{mz}$ & $12 q 9$ & 1099 & $10 \mathrm{qq}$ \\
\hline Valor leña por $\mathrm{mz}^{*}$ & 346.95 Lps. & 262.67 Lps. & $285.33 \mathrm{Lps}$. \\
\hline $\begin{array}{l}\text { Valor rastrojo por } \\
\mathrm{Mz}^{* *}\end{array}$ & $58 \mathrm{Lps}$. & $112 \mathrm{Lps}$. & I44 Lps. \\
\hline Valor tusa por $\mathrm{mz}^{*}$ & 44 Lps. & 49 Lps. & $73 \mathrm{Lps}$. \\
\hline $\begin{array}{l}\text { Costos en insumos/ } \\
\text { mz }\end{array}$ & 108.40 Lps. ${ }^{* *}$ & 1022.77 Lps. & 1139.31 Lps \\
\hline $\begin{array}{l}\text { Días trabajados por } \\
\text { mz ** }\end{array}$ & 61 dias & 57 días & 50 dias \\
\hline $\begin{array}{l}\text { Costos totales por } \\
\mathrm{mz}^{*}\end{array}$ & $3370.09 \mathrm{Lps}$ & 4383.49 Lps & $4261.01 \mathrm{Lps}$ \\
\hline $\begin{array}{l}\text { Ingreso del capital } \\
\text { por } \mathrm{mz} * *\end{array}$ & $63.69 \mathrm{Lps}$ & 178.72 Lps & $1151.78 \mathrm{Lps}$ \\
\hline $\begin{array}{lll}\text { Retorno mano } & \text { de } \\
\text { obra por } & \text { dia } \\
\text { trabajado } & & \\
\end{array}$ & 59 Lps & 64 Lps & $88 \mathrm{Lps}$ \\
\hline Relación $\mathrm{B} / \mathrm{C}$ & 1.10 & 1.08 & 1.32 \\
\hline
\end{tabular}

* Diferencias significativas para un rango de $95 \%$ de confidencia

* Diferencias significativo para un rango de $80 \%$ de confidencia

Figura 2. Indicador económico sobre el sistema Quesungual. Fuente Tejeda 2013.

En el cuadro anterior se puede observar como el Sistema Agroforestal Quesungual mejora no solo el ingreso de los personas sino también los días trabajados y nacionalmente el producto interno bruto.

Los sistemas agrícolas predominantes en el área del proyecto de PESA/ FAO son granos básicos (maíz, frijol, maicillo). Además existen cultivos de arroz, ajonjolí, sandia, caña de azúcar y variedades de musáceas . 


\section{Tipos de Cultivos}

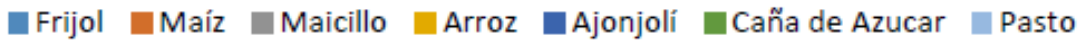

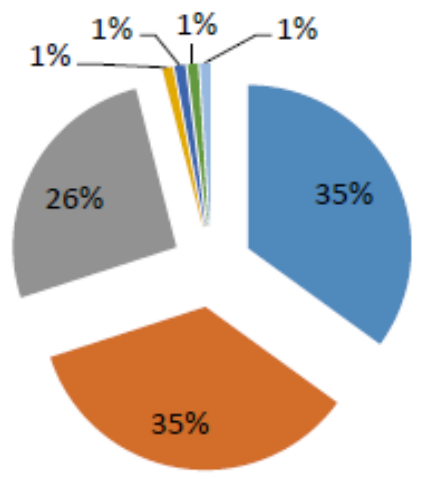

Elaboración propia

Grafico 1. De las 40 encuestas aplicadas a productores se puede apreciar que en la actualidad la mayoría de familias en la zona rural del Municipio de La Virtud produce maíz y frijol, en menor cantidad otros cultivos como: arroz, ajonjolí, caña de azúcar y pasto (toreño)

\section{- $\quad$ Caracterización de Tipos de Agricultura}

El uso inadecuado de la tierra ha sido un problema ambiental que ha afectado los suelos del Municipio de la Virtud, generando graves procesos de erosión, pérdida de fertilidad del suelo, desforestación, deterioro de los pastizales y la subutilización de la tierra de buena calidad, sin embargo, con la implementación del sistema Quezungual, la cual es una práctica tradicional agroforestal en la zona baja de Lempira implementada por PESA-FAO, a través de los años se ha aumentado la productividad de la parcela, se ha conservado la humedad del suelo, se ha creado soporte para la cosecha de maíz y frijol a través de las podas, en general se ha detectado recuperación del suelo desnudo, en donde hoy en día se ha dado reemplazo de vegetación .

FAO a través de PESA ha promovió tres tipos de agricultura: la de conservación, la orgánica y el manejo biológico integrado de los suelos, con el fin de sacar el máximo provecho a los sistemas de cultivos, siendo los principales cultivos agrícolas de la región de Lempira el maíz, frijol y café . (Figura 3) 


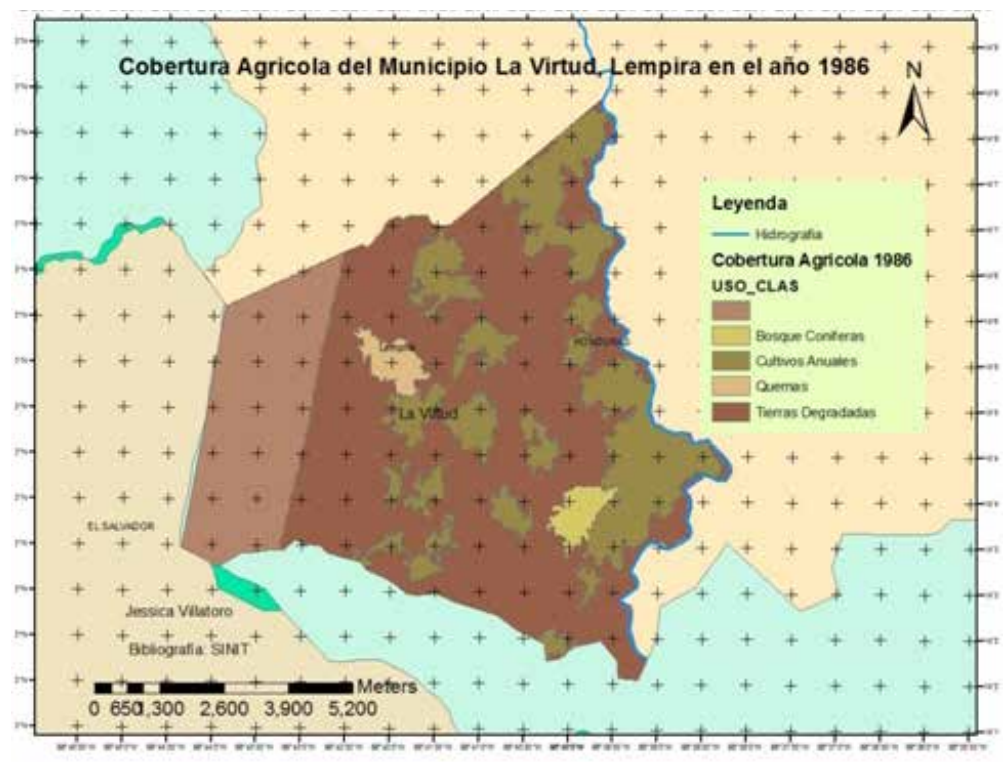

Figura 3. Cobertura Agrícola del Municipio de La Virtud, año 1986

En el año 1986 el área de cultivos anuales era menor, observando la existía predominante de tierras degradadas probablemente debido a malas prácticas agrícolas.

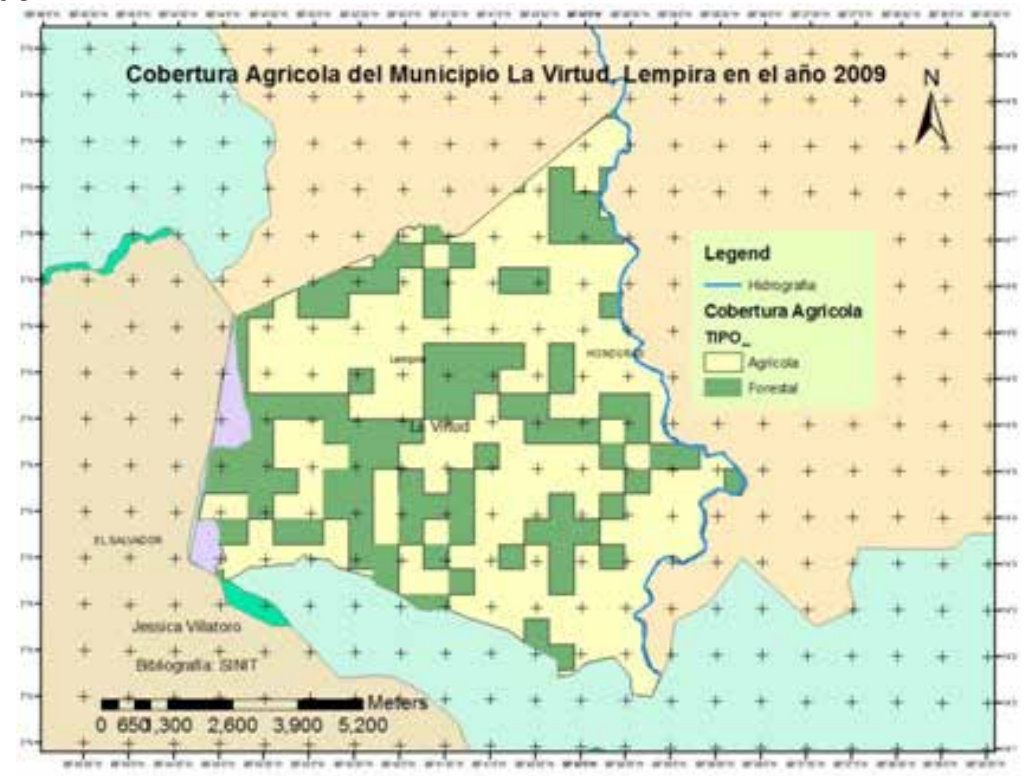

Figura 4. Cobertura agrícola del Municipio de La Virtud, año 2009 
A medida la población crece y mejora sus prácticas agrícolas, Observamos en el 2009 un aumento de la cobertura agrícola y forestal.

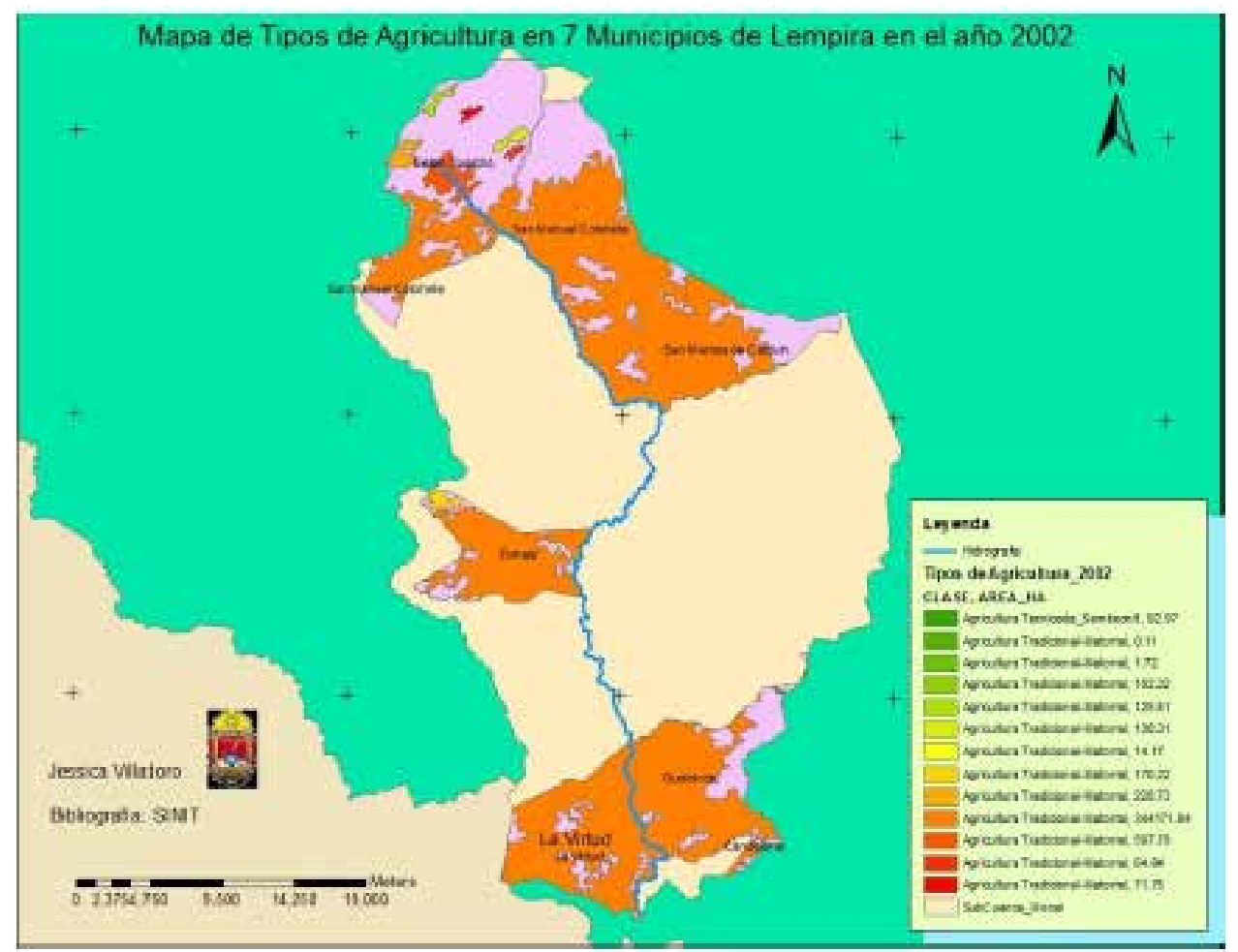

Figura 5. Tipos de Agricultura en la Subcuenca del Río mocal, año 2002

Comparando la Agricultura Tradicional-Matorral y Agricultura Tecnificada-Semitecnificada a través de la Subcuenca del río Mocal se puede decir según datos del SINIT que hasta el 2002 el tipo de agricultura en la parte alta, media y baja de la Subcuenca es de tipo Tradicional-Matorral, solo 92.97 Ha ubicada en la parte alta de la Subcuenca, en el Municipio de Belén Gualcho se práctica agricultura Tecnificada-Semitecnificada. 


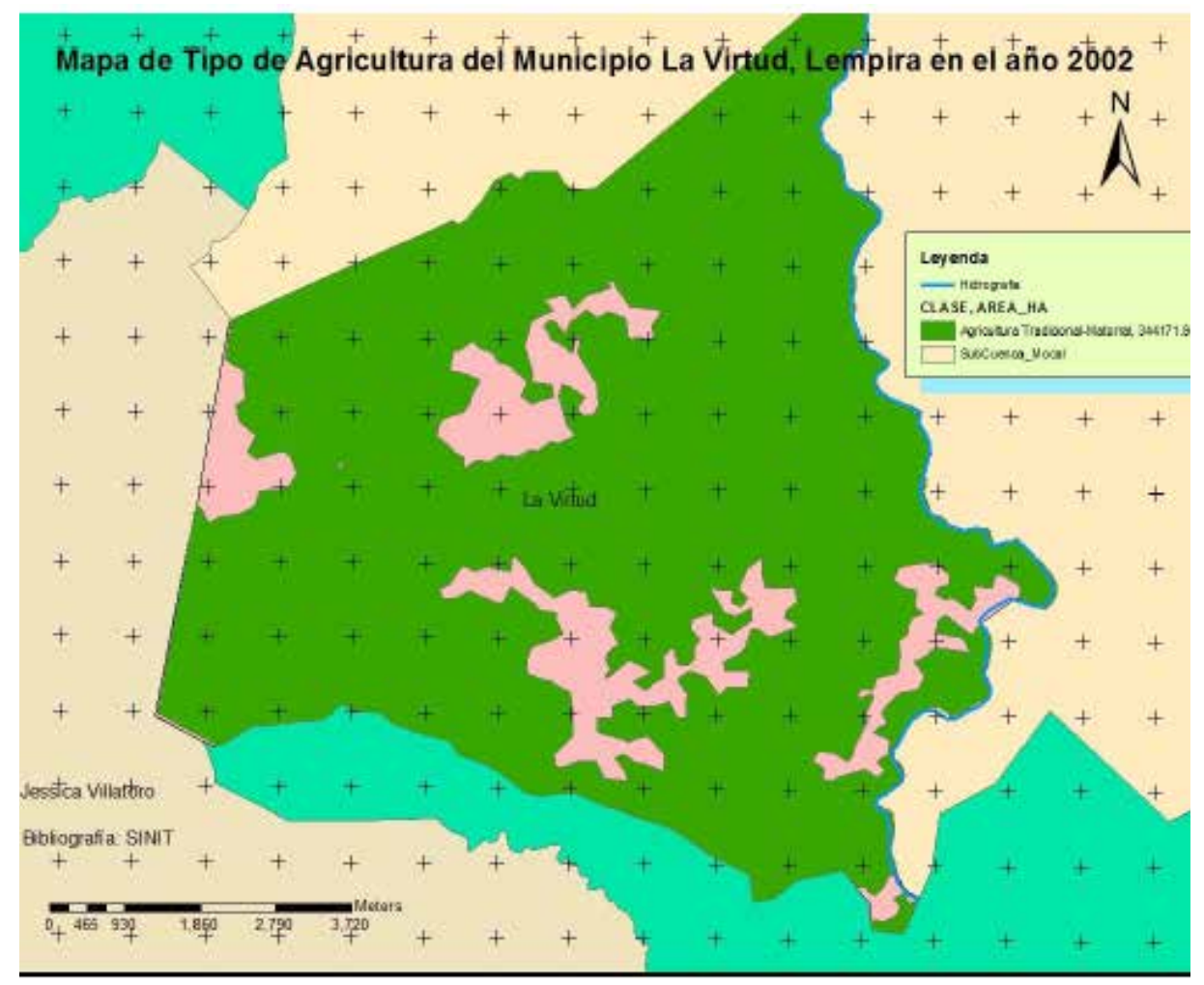

Figura 6. Mapa de tipo de agricultura del Municipio de La Virtud, año 2002

En el mapa se puede observar que en el año 2002 el tipo de agricultura que predomina en el Municipio de La Virtud es la Tradicional- Matorral.

\begin{tabular}{|c|c|c|c|c|c|}
\hline & & Frequency & Percent & Valid Percent & $\begin{array}{c}\text { Cumulative } \\
\text { Percent }\end{array}$ \\
\hline \multirow[t]{5}{*}{ Valid } & Muy mala & 2 & 5.0 & 5.0 & 5.0 \\
\hline & Regular & 13 & 32.5 & 32.5 & 37.5 \\
\hline & Buena & 23 & 57.5 & 57.5 & 95.0 \\
\hline & muy buena & 2 & 5.0 & 5.0 & 100.0 \\
\hline & Total & 40 & 100.0 & 100.0 & \\
\hline
\end{tabular}

Cuadro 1. Tipo de agricultura agroforestal 
La mayoría de personas encuestadas (57.5\%) perciben la agricultura agroforestal (quezungual) como buena, esto indica que aunque en el Municipio de La Virtud no todos la practican este tipo de agricultura, tienen claro que es una de las mejores alternativa para cuidar el suelo.

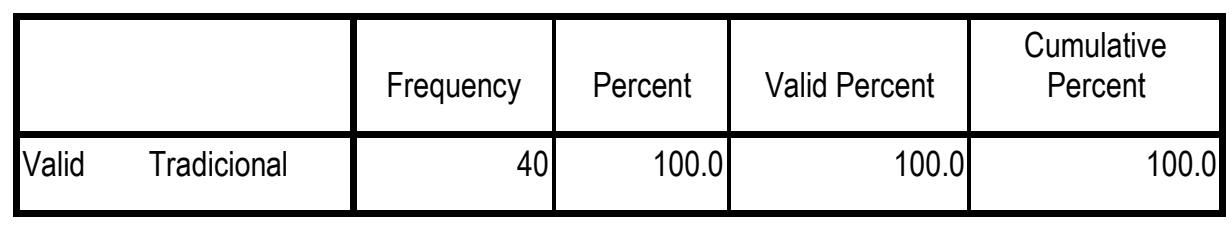

Cuadro 2. Tipo de Agricultura tradicional

Al preguntar qué tipo de agricultura se practicaba, se indicaron tres opciones: tradicional, agroforestal y silvopastoril, por tanto, según los resultados obtenidos vemos que en las comunidades de La Trinidad, Tixila y Solicor la mayoría de productores práctica la agricultura tradicional, misma que puede estar afectando la cobertura del suelo.

- $\quad \underline{\text { Clima: }}$

El clima en La Virtud, Lempira es de tipo lluvioso con invierno muy seco y se caracteriza por lluvias distribuidas de manera irregular entre mayo a diciembre. Los meses más lluviosos son junio y septiembre y los más secos: diciembre, enero y febrero. Las condiciones de clima permiten una amplia gama de especies de cultivos adaptadas a estas condiciones.

En las últimas décadas se está dando el fenómeno del cambio climático cuyos efectos son indiscutibles. Se estima que las emisiones de gases invernadero del pasado significaron calentamiento inevitable, aproximadamente incrementos de 1.6-2 grados centígrados a fin de siglo XX.

Se prevé fenómenos de fuertes precipitaciones, olas de calor en gran porcentaje de los ecosistemas terrestres y contrariamente, épocas de sequía en grandes áreas, facturando al sector agrícola especialmente por stress por calor, erosión, saturación hídrica, y otros. 


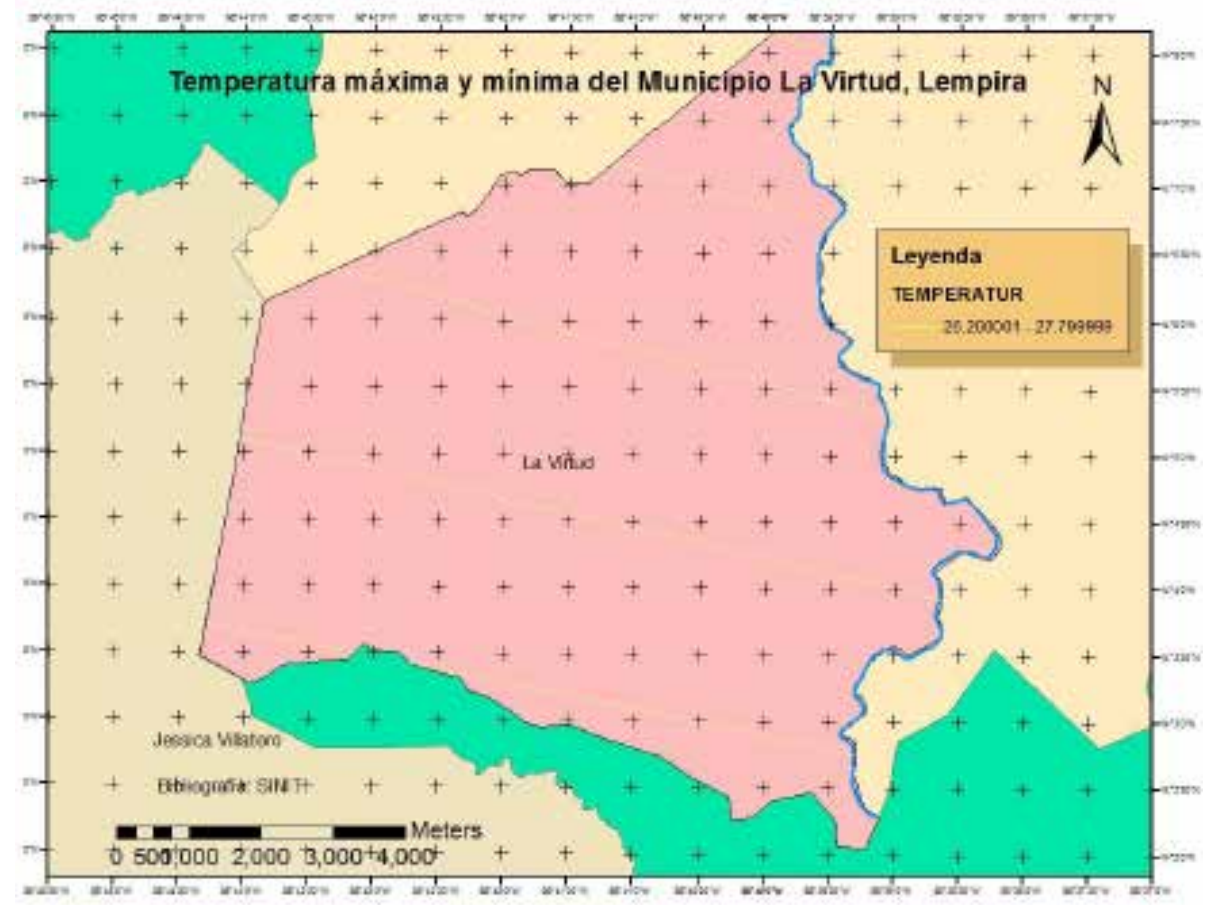

Figura 7. Temperatura máxima y mínima del Municipio de La Virtud

Con respecto al comportamiento de la temperatura, los datos registrados por el SINIT, muestran que la tendencia de la temperatura mínima en el Municipio de la Virtud es de 26 grados centígrados y máxima de 28 grados centígrados. Siendo uno de los Municipios más calientes de Lempira. 


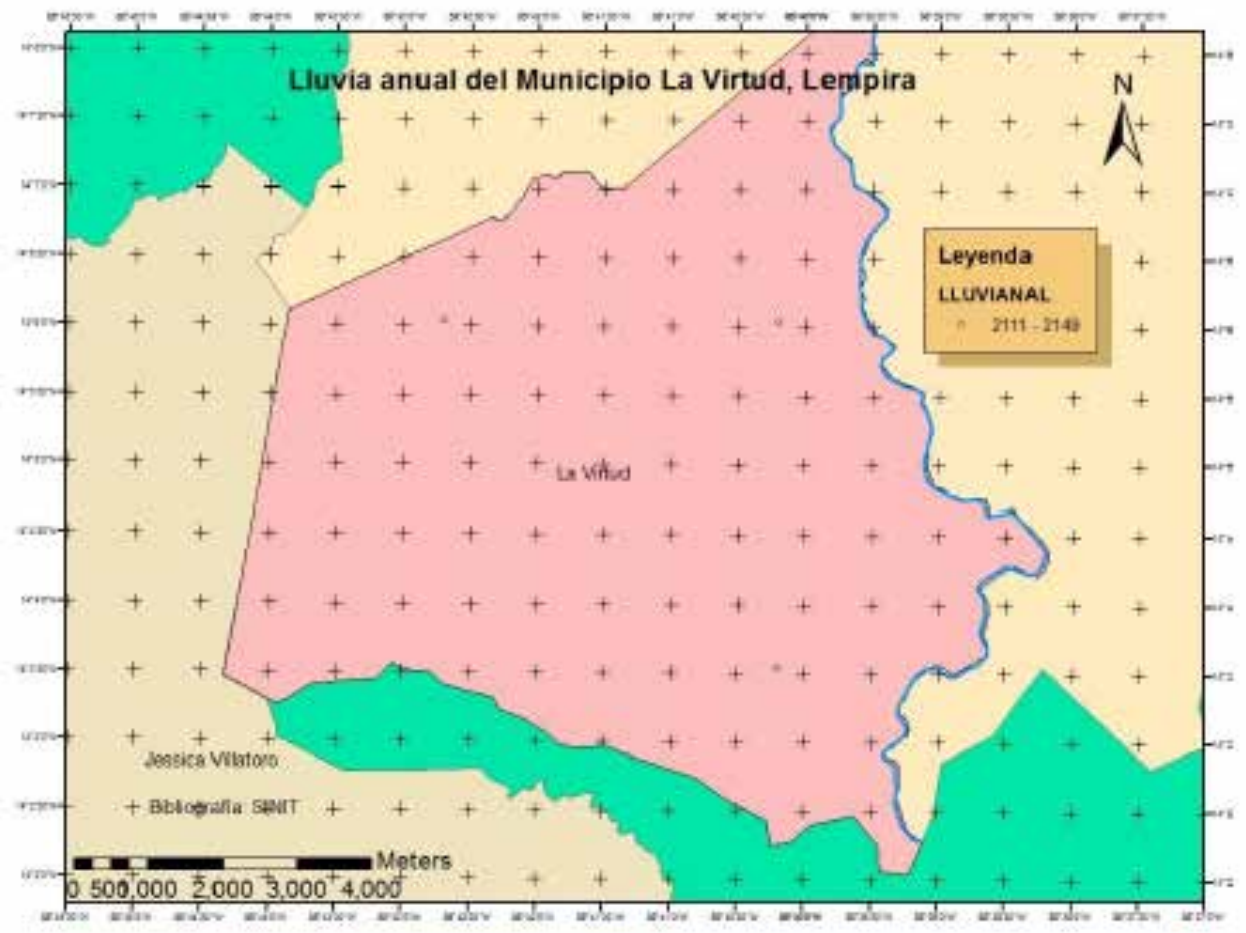

Figura 8. Lluvia anual del Municipio de La Virtud

Según datos del SINIT. La precipitación anual del Municipio de La Virtud varía entre 2111-2149 metros cúbicos, siendo los meses más lluviosos septiembre y octubre.

\section{Conclusiones}

1. La metodología empleada permitió conocer que actualmente productores del Municipio de La Virtud practican agricultura tradicional, misma que puede causar problemas en la cobertura de la tierra y en todo el medio ambiente.

2. Se puede determinar que en el Municipio de La Virtud se siembra maíz, frijol y maicillo, siendo la dieta básica de la mayoría de familias de la zona.

3. La caracterización del Municipio de La Virtud es solo el principio de un primer diagnóstico de la zona de la Subcuenca del río Mocal, lo que permitirá hacer un análisis más exhaustivo y analizar un estudio que indique los cambios de cobertura agrícola vrs. Los tipos de agricultura empleadas. 


\section{Bibliografía}

- Argeñal, F. J. (2010). Variabilidad Climática y Cambio Climático en Honduras. Tegucigalpa: PNUD.

- Benites, J. R., \& Álvarez Welchez, L. (1994). Ordenación Integrada de Recursos Naturales con Énfasis en Suelo, Agua y Nutrientes de Plantas. Candelaria: FAO.

- FAO. (16 de abril de 2005). Depósitos de FAO, Departamento de Agricultura. Obtenido de Agricultura y Desarrollo Rural Sostenible: http://www.fao.org/docrep/meeting/009/j4236s/j4236s00.HTM

- Fernández, Liliana; Navarro, Edgardo; FAO. (2005). El Sistema Agroforestal Quesungual. Tegucigalpa: Litografía López.

- Nájera, Bojórquez, Lemus, Marceleño. (2010). Cambio de cobertura y uso del suelo en la cuenca del río Mololoa, Nayarit. Biociencias, 29.

- Tejeda, M. J. (18 de agosto de 2013). "HONDURAS SI SE PUEDE" Asegurar sus alimentos cambiando sus sistemas de producción tradicionales en laderas. Obtenido de http://mildred-tejada.rds.hn/honduras_si_puede.pdf 